\title{
A la parrilla suena mejor: El podcast como estrategia didáctica basada en el ABP para el análisis y la gestión de eventos en la asignatura Historia de la Música
}

\author{
A la parrilla suena mejor: Podcast as a Project-Based- \\ Learning Didactic Tool for Music History and Event \\ Management
}

\author{
Diego García-Peinazo \\ digarpei@gmail.com \\ Universidad de Córdoba, España
}

$\begin{array}{ll}\text { Recibido } 17 / 10 / 2018 & \text { Revisado 10/11/2018 } \\ \text { Aceptado 19/11/2018 } & \text { Publicado 01/01/2019 }\end{array}$

\section{Resumen}

Este artículo expone diversas aplicaciones didácticas del podcast para la enseñanza y aprendizaje de la asignatura Historia de la Música en el ámbito universitario. Concretamente, se fundamenta su utilidad como herramienta para el estudio, análisis y gestión de eventos musicales preexistentes -festivales, ciclos de conciertos, etc.- con el alumnado de Grado de Gestión Cultural. Por medio de este recurso radiofónico, que favorece el Aprendizaje Basado en Proyectos

\section{Abstract}

This paper deals with different didactic strategies based on podcast in the teachinglearning context of the subject Music History in Graduate University Education. Specifically, podcast is presented here as a tool for the study, analysis and management of pre-existent music events -festivals, musical projects, etc.- with students of the BA in Cultural Management (Universidad de Córdoba). Through this tool, which facilitates the Project-Based-Learning (PBL) process, the students can understand,

Sugerencias para citar este artículo García-Peinazo, Diego (2019). A la parrilla suena mejor: El podcast como estrategia didáctica basada en el ABP para el análisis y la gestión de eventos en la asignatura Historia de la Música. Tercio Creciente, 15, págs.73-84. https://dx.doi.org/10.17561/rtc.n15.5

GARCÍA-PEINAZO, DIEGO. A la parrilla suena mejor: El podcast como estrategia didáctica basada en el ABP para el análisis y la gestión de eventos en la asignatura Historia de la Música. Tercio Creciente, enero 2019. $\mathrm{n}^{\circ}$ 15, pp. 73-84. https://dx.doi.org/10.17561/rtc.n15.5 
(ABP), el alumnado conoce, examina y experimenta con los repertorios de música académica que forman parte de los contenidos de la asignatura, y descubre cómo éstos son gestionados por parte de instituciones y organismos públicos y privados, aspectos fundamentales en la formación de los futuros profesionales de la gestión cultural. En primer lugar, este trabajo aborda la didáctica de la historia de la música desde una dimensión crítica que contempla tanto aspectos teóricometodológicos como de adaptación curricular a la titulación superior en la que se imparte y se fundamenta la idoneidad del podcast como herramienta del APB en el ámbito de la educación musical. Tras ello, se detallan las características del proyecto planteado, desarrollado en dos fases: fase de análisis y diagnóstico; fase de difusión y promoción. Por último, se evidencia la utilidad didáctica del podcast para el gestor cultural, por un lado, como medio para vivenciar la historia de la música y, por otro, como fin, en tanto herramienta útil para difundir y comunicar aspectos relativos a eventos musicales. experience and examine different Western Art Music repertoires, and the way these repertoires are mediated and managed by a range of institutions and organizations as crucial elements in the learning process of future professionals of cultural management. In a first step, this article attends to some aspects regarding the pedagogy of music history from a critical point of view, considering both theoretical framework and the syllabus associated with the degree in Cultural Management, dealing also with the importance of podcast and PBL in music education. It is exposed the main characteristics of the project A la parrilla suena mejor, which is developed in two steps: analysis and diagnosis; broadcasting and promotion. Furthermore, I discuss about the didactic importance of podcast in order to understand music histories as well as a professional tool in order to communicate ideas related to musical events.

\section{Palabras clave / Keywords}

Historia de la Música; Didáctica de la Expresión Musical; Podcast; Educación Superior; Aprendizaje Basado en Proyectos (ABP); Gestión Cultural; Eventos musicales.

Music History; Music Education; Podcast; Graduate (University) Education; Project Based Learning (PBL); Cultural Management; Musical Events

Sugerencias para citar este artículo

García-Peinazo, Diego (2019). A la parrilla suena mejor: El podcast como estrategia didáctica basada en el ABP para el análisis y la gestión de eventos en la asignatura Historia de la Música. Tercio Creciente, 15, págs.73-84. https://dx.doi.org/10.17561/rtc.n15.5

GARCÍA-PEINAZO, DIEGO. A la parrilla suena mejor: El podcast como estrategia didáctica basada en el ABP para el análisis y la gestión de eventos en la asignatura Historia de la Música. Tercio Creciente, enero 2019. $\mathrm{n}^{\circ}$ 15, pp. 73-84. https://dx.doi.org/10.17561/rtc.n15.5 


\section{Historias de la música en su (con)texto: del currículo educativo español al Grado de Gestión Cultural}

La asignatura de Historia de la Música tiene una presencia variable en diferentes etapas educativas y tipologías de estudios. Durante la Etapa Primaria y en mayor medida en la Etapa de Enseñanza Secundaria Obligatoria, la asignatura Música aborda contenidos relativos a la historia de la música académica occidental, si bien es en itinerarios específicos del Bachillerato, como el de la rama de Artes Escénicas, Música y Danza o en la de Humanidades, donde se hallan asignaturas específicas sobre la disciplina. En las denominadas Enseñanzas de Régimen Especial, esto es, conservatorios y escuelas de música, aparece como asignatura obligatoria en los cursos $4^{\circ}$ y $5^{\circ}$ de las Enseñanzas Profesionales, mientras que en $6^{\circ}$ curso se imparte Historia del Pensamiento Musical $^{1}$. En el caso de los Estudios Superiores de conservatorio, se encuentra en los planes de estudio tanto de especialidades de interpretación como en las de pedagogía, composición, dirección, etc. con varias asignaturas troncales, además de conformar uno de los pilares formativos de la especialidad de Musicología. En el ámbito universitario ${ }^{2}$, además ser parte constitutiva de los Estudios de Grado de Historia y Ciencias de la Música, está presente de manera intermitente en grados vinculados a la rama o macro-área de Artes y Humanidades en forma de asignaturas obligatorias, como en el Grado de Historia del Arte $^{3}$.

Historia de la Música se imparte con carácter obligatorio en el tercer curso de
Grado de Gestión Cultural de la Universidad de Córdoba. La formación en materia musical ofrecida en el grado se completa gracias a asignaturas como Lenguajes Artísticos: Introducción al lenguaje de la Música y a los lenguajes de la fotografía y el cine (segundo curso), o Gestión y Organización de Eventos y Actividades Musicales y Escénicas (cuarto curso), ambas obligatorias. La orientación de una asignatura dedicada al estudio de la historia de la música en este grado requiere de un enfoque que prime una dimensión crítica de la acción del hecho sonoro en las esferas social, económica, política y cultural desde la Antigüedad al siglo XXI, en consonancia con los postulados emprendidos en los años ochenta del siglo XX por la denominada Nueva Musicología, así como por la Historia Cultural de la Música (Fulcher, 2011). Sin embargo, es igualmente preciso que el alumnado conozca aspectos relativos a compositores, obras, géneros y repertorios, todos ellos revisados críticamente por dichas corrientes musicológicas, pero que forman parte indisoluble de las programaciones en eventos musicales.

En último término, todo proceso de selección de obras y compositores que merecen ser nombrados e historiados en las aulas es, al fin y al cabo, un ejercicio de inclusión y no-inclusión que se vincula a la construcción de un canon musical. Este puede evidenciarse, por ejemplo, a través 
de antologías de partituras (Bonds, 2011), en la propia trayectoria histórica de la pedagogía de las músicas populares urbanas, que ha tendido a nuevos procesos de canonización en lugar de a la audición activa (García-Peinazo, 2017), o por medio de la construcción discursiva de dicho canon en los libros de texto educativos en España, que acarrean cuestiones de ideología, género y modelo cultural, siendo un referente sobre estas cuestiones las investigaciones Berta Pérez-Caballero Rubio (2017 y 2018). Así, uno de los puntos clave indicados por Lisa C. DeLorenzo acerca de la docencia de la música como práctica democrática apela a la reflexión en torno a si empleamos en las aulas tanto música del canon reconocido como de compositores y compositoras subalternos (2003, p. 38).

En nuestra asignatura, dada la orientación hacia las músicas académicas occidentales y la mencionada necesidad de trabajar con los conceptos de canon, autor y obra, establecemos dos actividades, una de iniciación y otra de consolidación, que pretenden cuestionar la inmutabilidad de los repertorios de la historia de la música y evidenciar dicho canon, dado que, aunque el alumnado ha de conocer qué repertorios son los más frecuentes en las programaciones, también se precisa que el futuro gestor o gestora sea consciente de los procesos culturales implicados detrás de dicho canon. Por una parte, en la sesión inicial de la asignatura se plantea al alumnado la actividad de inicio "Golden Record y la historia de la música”, en la cual, en analogía a la grabación sonora Golden Record enviada junto con la Sonda Voyager en 1977, se les solicita que elijan un track que consideren más importante y representativo, desde su criterio como gestores, para ser enviado al espacio, requiriendo que sus respuestas se fundamenten en cualquier tipo de criterio, ya sea musical, social, cultural, económico, histórico, etc. De esta forma, la disparidad de tracks y fundamentos de "rescate" para conformar un "patrimonio históricoterráqueo" evidencia que toda conformación de repertorios en la historia de la música, incluida la del propio docente, es excluyente y parcial. Por otro lado, la actividad de consolidación, que se lleva a cabo de forma paralela al proyecto del podcast, consiste en la elaboración de una lista compartida -puede emplearse la multiplataforma Spotify o similar- en la cual cada alumno o alumna selecciona una obra relativa a la música académica y fundamenta por qué ha de ser parte del listado de audiciones objeto de estudio para la resolución de una de las preguntas del ítem de evaluación de la prueba escrita. Aunque estas dos actividades no se vinculan, sensu stricto, al proyecto del podcast dedicado a eventos musicales, permiten llevar a cabo una tormenta de ideas de carácter reflexivo sobre la forma en que se ha narrado la historia de la música -en el caso de la actividad de inicio- y facilitan la experimentación de dicha construcción del repertorio a partir de sus propios criterios selectivos -actividad de consolidación-.

\section{Eventos musicales, podcasts y Aprendizaje Basado en Proyectos (ABP)}

El podcast ha sido empleado como eje vertebrador de experiencias centradas en el Aprendizaje Basado en Proyectos (ABP) orientado a las TICS:

El ABP debe ser una de las herramientas fundamentales para el desarrollo de las competencias (creatividad, resolución de problemas, habilidad de investigar, trabajar de forma colaborativa, motivación y uso de redes 
sociales, entre otras) que se van a potenciar en los sistemas educativos del siglo XXI (...) el uso del podcast mediante la metodología ABP en el ámbito universitario (...) facilita el aprendizaje autónomo del estudiante, a su propio ritmo, así como fomenta la participación activa y el aprendizaje. (Ausín et al. 2016, p. 32)

Así, su uso como herramienta para favorecer el proceso de enseñanza y aprendizaje en el ámbito universitario ha sido constatado por trabajos como el de Ramos García y Caurcel Cara (2011), además de favorecer procesos de democratización, "edición libre y horizontal de la información” en el ámbito educativo (Solano Fernández, Sánchez Vera, 2010). En el caso de la educación musical, experiencias didácticas como la expuesta por Ignacio Climent en el CEFIRE de Xàtiva (Valencia) evidencian que el podcast permite un abordaje diferencial de los contenidos de la asignatura de música, favoreciendo "el trabajo transversal y competencial con nuestro alumnado" (Climent 2018, p. 39). De la misma forma, independientemente del uso de una u otra herramienta asociada a las TICS, desde la propia gestión profesional de festivales y ciclos de conciertos pueden articularse propuestas didácticas significativas, como demuestran los trabajos al respecto de Javier Marín López y Virginia Sánchez López (2009) a propósito de los conciertos didácticos del Festival de Música Antigua de Úbeda y Baeza, o de Miguel Ángel Marín e Isabel Domínguez (2015) en relación a los conciertos didácticos de la Fundación Juan March.

Siguiendo a Kerstetter (2009, p. 26), el podcast es un recurso de fácil implementación en el aula de música, presentando una flexibilidad que facilita al docente la posibilidad de alcanzar una gran cantidad de objetivos. La idoneidad de una herramienta como el podcast en la asignatura "Historia de la Música” del Grado de Gestión Cultural radica en sus posibilidades de comprensión, análisis, organización y gestión de eventos musicales. El podcast se convierte, por un lado, en un medio para conocer los repertorios más programados en el marco de eventos musicales organizados en la actualidad en España. Por otra parte, en manos del alumnado, futuros profesionales de la gestión cultural, se torna un fin, ya que a través del archivo radiofónico elaborado por ellos y ellas aprenden a difundir y promocionar eventos desde el punto de vista mediático interiorizando, al mismo tiempo, contenidos relativos a las músicas académicas occidentales desde la Antigüedad al siglo XXI. De esta forma, se atiende tanto a competencias transversales que fomentan el trabajo en equipo, el conocimiento autónomo y la dimensión creativa en la elaboración de contenidos radiofónicos, como competencias profesionales del ámbito de la gestión cultural.

\section{A la parrilla suena mejor: objetivos y fases del proyecto}

A continuación, se presenta una descripción del proyecto A la parrilla suena mejor, el cual fue planteado para el alumnado de tercero de Grado de Gestión Cultural (Universidad de Córdoba) durante el curso $2017 / 2018$, y cuyo objetivo fundamental fue el estudio, análisis, diagnóstico y promoción de eventos musicales existentes en España mediante la elaboración cooperativa de 
un podcast. A través de este el alumnado experimenta, de forma práctica, los contenidos relativos a los diferentes periodos de la historia de las músicas académicas occidentales y, por otro, descubre y examina críticamente algunos de los festivales, ciclos de conciertos y demás eventos de mediano o gran formato que se llevan a cabo en el país.

El proyecto se presenta la primera semana de clase y se desarrolla a lo largo de todo el cuatrimestre, en el cual se destinan diversas sesiones teórico-prácticas (desde el análisis de repertorios musicales hasta la explicación de los rudimentos técnicos del software Audacity), así como tutorías grupales, además del trabajo autónomo por grupos. Si bien el proyecto podría plantearse en términos de una historia de los eventos musicales en España a través del estudio comparativo de diferentes temporadas, se sugiere al alumnado que centre su análisis en aquellos eventos desarrollados en la temporada inmediatamente anterior a la del curso académico en la que se lleva a cabo el proyecto. Éste se articula, tras la distribución de la clase en grupos de entre tres y cinco personas, en dos fases principales: en primer lugar, una fase de análisis y diagnóstico y, en segundo, una fase de difusión y promoción.

\section{Fase de análisis y diagnóstico}

En su primera fase, el proyecto consiste en el análisis y diagnóstico de un evento preexistente desarrollado en España relacionado con alguno de los periodos de la historia de la música occidental abordados en clase desde el punto de vista de la gestión cultural. Dicho evento ha de referirse necesariamente a "músicas académicas" (un término no exento de controversia, pero más "desideologizado", "neutro" y "preciso" que el de músicas cultas, históricas o clásicas) desde la Antigüedad hasta el siglo XXI. El objetivo fundamental del trabajo, en esta fase, es conocer la manera en que la historia de la música occidental es gestionada desde políticas culturales, instituciones y organismos públicos y privados, así como familiarizarse con los principales elementos que componen los festivales de estas características. De esta forma, durante esta fase, los grupos recaban información para la selección de un evento musical -festival, ciclo de conciertos, etc.- acaecido en los últimos años. Cada grupo consensua con el docente qué evento elegirá y las razones de dicha selección. Se sugiere al alumnado que, antes de su elección, tantee las posibilidades de acceso y el volumen de información disponible en los portales web oficiales de los propios eventos, ya que esto condicionará la realización del proyecto. No obstante, la ausencia de información también permite al alumnado un aprendizaje significativo a través del establecimiento de puntos fuertes y deficiencias en la difusión y la promoción de eventos. Tras la elección, el alumnado ha de buscar información en cartelería, programaciones, programas de mano, consulta a instituciones y organismos diversos, web oficial y otros portales web, etc., referida a los siguientes puntos:

1) Análisis de elementos históricomusicales en relación con el cartel/ programación: periodo de la historia de la música occidental en el que se centra; mención a instrumentos musicales; mención a estilos/géneros/ formas musicales; Información sobre compositores y obras musicales; Información sobre intérpretes musicales; Inclusión de repertorio musical de compositores españoles; Relación con el público/ audiencias; Referencia al contexto sociocultural y político. 
2) Análisis de elementos relacionados con la gestión del evento: Resumen/ descripción del proyecto; finalidades del proyecto; organización y profesionales de la música implicados; patrocinio; financiación pública y/o privada; nicho de mercado/ audiencias potenciales; presupuesto/ memoria económica; precios; espacios empleados; fechas (época del año y duración), días y horas; presencia en redes sociales; vías/medios de promoción y difusión; publicaciones discográficas/bibliográficas asociadas.

\section{Fase de difusión y promoción}

Esta segunda fase del proyecto A la parrilla suena mejor atiende a la creación y diseño de un programa radiofónico relativo al evento analizado, en el cual se difunda y promocione el mismo, así como su posterior exposición en clase. El objetivo fundamental de esta fase es desarrollar una propuesta creativa relativa a la gestión cultural de un evento preexistente vinculado a la historia de la música occidental, así como familiarizarse con los principales elementos que componen los festivales de estas características y transferir los conocimientos, habilidades y actitudes aprendidos en el ámbito de la gestión a la historia de la música. Todo ello se enriquece a través del trabajo grupal, cuyos resultados son fruto del intercambio de ideas, la discusión, la resolución de conflictos y el aprendizaje cooperativo.

Los grupos han de realizar, en primer lugar, una memoria escrita del podcast, en la cual se detallan una contextualización y destinatarios, y se incluyen aspectos relativos al contexto sociocultural de la ciudad/municipio al que va dedicado el podcast. Se indica a los grupos que la audiencia potencial ha de ser preferentemente alumnado universitario de grados de Artes y Humanidades. Por otro lado, se solicita que el alumnado vaya recogiendo todo lo referido al proceso de elaboración del podcast en el "libro del evento" sus procesos de planificación inicial -reparto de tareas, toma de decisiones, recursos, espacios, etc.- como la elaboración del mismo. El alumnado ha de elaborar, además, un guion radiofónico en el cual se incluyan tanto los diálogos como los tracks seleccionados para ser incorporados al podcast. Aunque la estructura del podcast se deja a elección del alumnado, éste ha de contemplar tanto aspectos histórico-musicales vinculados a la programación como características de la gestión del evento.

La grabación del podcast, que ha de presentar una duración aproximada de entre 10 y 12 minutos, se lleva a cabo mediante el software libre Audacity. Dicha grabación se realiza, por parte de cada grupo, tras una sesión en la cual se explican algunos de los rudimentos en la edición de audio con este programa. Como directrices, se solicita al alumnado que la cantidad de música grabada incorporada al podcast sin ninguna intervención de voz hablada -esto es, no empleada como música de fondo- no exceda los 4 minutos. Para la selección de fragmentos musicales de audiciones, el alumnado ha de incorporar obras presentes en las programaciones de los eventos que ha analizado en la fase anterior y, a ser posible, de patrimonio musical español. Esto permite, por un lado, que el alumnado trabaje mediante la selección auditiva de fragmentos que considere relevantes y, por otro, descubre que obras del patrimonio musical español no están aún grabadas, teniendo que recurrir a grabaciones de actuaciones en directo a partir de plataformas como YouTube. Además, el trabajo con la selección de repertorios musicales pone al alumnado en contacto tanto con la noción de compositor e 
intérprete en música académica, aprendiendo a diferenciarlas, así como a conocer las industrias culturales vinculadas al hecho musical, desde la discográfica a la publicidad.

Como se mencionó con anterioridad, se pide que cada grupo intercale en sus podcasts fragmentos sonoros de audiciones con partes habladas, en las que participan todos los miembros del grupo. Por ello, se explican algunas estrategias para llevar a cabo estos aspectos con un resultado sonoro acorde al contexto de la radio. Entre otras, además de la edición básica a través de la importación y exportación de audio, la selección y recorte de fragmentos, ecualización, la configuración de las pistas o el paneo del canal izquierdo o derecho de las mismas, se recurre a la herramienta "envolvente". Esta permite modificar puntualmente y de forma no abrupta del volumen de una pista para facilitar así la escucha de una pista de voz hablada. De la misma forma, el fade in (aparición progresiva del sonido) y el fade out (desaparición progresiva del sonido) son trabajados en clase, tanto desde el punto de vista procedimental como desde una dimensión histórica, explicando la importancia de esta técnica en la historia de la programación radiofónica, desde lo analógico a lo digital.

Como cierre de esta última fase, cada grupo comparte en una exposición de veinte minutos frente al resto de la clase las características principales de los eventos musicales seleccionados. Dicha exposición implica la interiorización de un rol en el cual los alumnos y alumnas se convierten en protagonistas de la gestión en la cual se intercambian ideas y se conocen fortalezas y problemáticas compartidas entre estos eventos musicales de pequeño o gran formato. El mismo día de la exposición de cada grupo se lleva a cabo una coevaluación desde diferentes planos, tanto del gran grupo clase a cada uno de los grupos pequeños, como de los diferentes miembros del grupo al resto de sus compañeros de equipo, lo cual permite vivenciar, a su vez, la trascendencia de una organización y gestión eficaz entre los recursos humanos de un proyecto de este tipo.

\section{Epílogo: gestionar las historias de la música en el aula}

Según Climent, "la implantación del podcast en el proceso de enseñanzaaprendizaje requiere un proceso de reflexión, de justificación y de planificación adecuado" (2018, p. 41). A nuestro juicio, el proyecto $A$ la parrilla suena mejor, vinculado a la asignatura "Historia de la Música”, de tercero de Grado de Gestión Cultural, consigue dotar al alumnado de competencias transversales y profesionales de cara a la gestión de un evento musical preexistente mediante su concreción en la elaboración de un podcast. Algunas de las reflexiones y conclusiones del alumnado reflejan la utilidad de la herramienta para su formación como gestores culturales:

El uso del podcast confirma la potencia de este recurso como proceso de formación y transmisión de la información, en este caso de un festival de música académica preexistente en el panorama nacional español, además de beneficiar positivamente el aprendizaje de los estudiantes para posteriores trabajos (Grupo de clase $G, 2018$ ) (...) Las integrantes del grupo han tomado contacto con programas de edición de sonido. Este ha sido un soporte de difusión totalmente novedoso hasta ahora, ya que, hasta hace poco, solo dominábamos el formato de flyers informativos, tarjetas de visita o carteles publicitarios. Por otro lado, también nos 
hemos introducido en el mundo de la radio, conociendo los programas musicales que se encuentran inmersos en la emisora de música académica por excelencia, Radio Clásica. (Grupo de clase D, 2018)

Asimismo, se valoraron las dificultades y fortalezas en la elaboración del podcast a través de Audacity:

La elaboración del podcast ha sido algo laboriosa, ya que nunca habíamos trabajado con el programa Audacity, pero al tratarse de un programa muy intuitivo, finalmente hemos podido trabajar con él sin problema (Grupo de clase B, 2018) (...) Es nuestra primera toma de contacto con programas radiofónicos y de montaje de sonido. Se ha intentado realizar un podcast sencillo con la única finalidad de llegar a los oyentes de una forma clara, sintetizando ideas e informando de las diferentes actividades y conciertos que se desarrollarán, así como el precio de las entradas, descuentos a los jóvenes y los espacios elegidos para la realización del mismo (...) la elaboración del guion nos ha resultado sencilla ya que previamente teníamos la idea de realizar el podcast como si se tratara de un programa radiofónico sobre música en forma de entrevista. (Grupo de clase A, 2018)

Por último, el podcast fue descrito en términos de un instrumento para conocer las características principales en la gestión de un evento musical, el que atañe a "la promoción y difusión radiofónica” (Grupo de clase C, 2018), aunque también para registrar algunas limitaciones, por la complejidad de acceso a la información de algunos ítems solicitados para la Fase de Análisis y Diagnóstico del evento: sugerencias de este festival tan ecléctico y que aún hoy sigue salvaguardando temáticas de gran interés público, incorporando herramientas innovadoras como pueden ser instrumentos de época o la inclusión de otros estilos más conocidos entre la juventud. (Grupo de clase G, 2018)

Con este proyecto, desarrollado en tercer curso, se sientan las bases para que, de manera prospectiva, en los bloques dedicados a música de la asignatura Gestión y Organización de Eventos y Actividades Musicales y Escénicas, impartida en cuarto curso, el alumnado pueda llevar a cabo la producción y difusión de un evento musical diseñado por ellos mismos. En este otro proyecto el abanico de repertorios no se reduce a la música académica occidental, sino que se abre a la multitud de músicas que abarcan macro etiquetas como músicas populares urbanas, músicas tradicionales y las citadas músicas académicas, desarrollando con el alumnado metodologías específicas para atender al impacto cultural y mediáticos de eventos musicales en la gestión del municipio.

En síntesis, el proyecto A la parrilla suena mejor pretende situar al alumnado y futuro profesional de la gestión cultural en uno de los puntos centrales del hecho musical. Si ellos son una parte importante del proceso de construcción de las historias cotidianas de la música, como alumnos/as con prácticas diarias de consumo musical y juicios valorativos sobre música, serán también futuros profesionales de la cultura que jugarán un papel destacado gestionando la historia de la música como programadores de repertorios y prácticas musicales concretas.

Gracias al uso del podcast se ha podido dilucidar las fortalezas, debilidades y 


\section{Notas}

1. Orden de 25 de octubre de 2007, por la que se desarrolla el Currículo de las Enseñanzas Profesionales de Música en Andalucía (Anexo III, p. 194)

2. Un estado de la cuestión de la enseñanza de la música a nivel internacional se encuentra en Mary Natvig (2002). Asimismo, cabe destacar, como hitos importantes en la disciplina, la existencia de revistas científicas dedicadas a la didáctica de la historia de la música, como el Journal of Music History Pedagogy (desde 2010), así como la constitución de la rama de educación musical vinculada a la International Musicological Society (IMS) (2018).

3. La asignatura de "Historia de la música" presenta una relativa consolidación en el Grado de Historia del Arte de diversas universidades españolas, contando incluso con manuales de estudio, como el de González Martínez (2015). En relación a los manuales dedicados a la materia de "Historia de la música” en enseñanzas profesionales de música, véase, por ejemplo, el trabajo de Pajares Alonso (2014).

4. Empleamos aquí el término "libro del evento" siguiendo el trabajo de Turbau (2010).

\section{Referencias}

Ausín, Vanesa; Abella, Víctor; Delgado, Vanesa; Hortigüela, David (2016). Aprendizaje Basado en Proyectos a través de las TICS. Una Experiencia de Innovación Docente desde las Aulas Universitarias. Formación Universitaria, 9/3, pp. 31-38. Doi: 10.4067/S071850062016000300005

Bonds, Mark Evan (2011). Selecting Dots, Connecting Dots: The Score Anthology as History. Journal of Music History Pedagogy, 1/2, pp. 71-91.

DeLorenzo, Lisa C. (2003). Teaching Music as Democratic Practice. Music Educators Journal, 90/2, pp. 35-40. Doi.org/10.2307/3399932

Fulcher, Jane F. (ed.)(2011). The Oxford Handbook of The New Cultural Historyof Music. New York: Oxford University Press. 
ISSN: 2340-9096

DOI: https://dx.doi.org/10.17561/rtc.

https://dx.doi.org/10.17561/rtc.n15.5

García-Peinazo, Diego (2017). ¿Nuevos “clásicos básicos” en educación musical? De la canonización a la audición activa de las músicas populares urbanas en (con)textos didácticos específicos. LEEME: Revista Arbitrada de Investigación y Aplicaciones en Educación Musical, 40, pp. 1-18. Doi.org/10.7203/LEEME.40.10914

González Martínez, Juan Miguel (2015). La música en la historia del arte: materiales didácticos. Murcia: Universidad de Murcia.

IMS Study Group “Transmission of Knowledge” (2018). International Musicological Society (IMS), http://www.ims-education.net/home/about-us/ [Consulta 28/09/2018].

Journal of Music History Pedagogy (2002). AMS, http://www.ams-net.org/ojs/index.php/jmhp/ [Consulta: 29/09/2018].

Kerstetter, Kathleen (2009). Educational Applications of Podcasting in the Music Classroom. Music Educators Journal, 2009, 95/4, pp. 23-26. Doi.org/10.1177/0027432109334691

Marín, Miguel Ángel y Domínguez, Isabel (2015). Aprender por objetivos: un modelo de conciertos didácticos en la Fundación Juan March. Eufonía: Didáctica de la Música, pp. 29-38.

Mary Natvig (ed.) (2002). Teaching Music History. London: Routledge.

Pajares Alonso, Roberto L. (2014). Historia de la Música para Conservatorios (en dos volúmenes), Madrid: Visión Libros.

Pérez-Caballero Rubio, Berta (2017). Libros de texto, o “todo” lo que debes saber sobre música. DEDiCA. Revista de Educaçâo y Humanidades, 11, pp. 133-149.

Pérez-Caballero Rubio, Berta (2018). Canon musical y modelo cultural en la educación secundaria obligatoria a partir de la LOGSE. Tesis doctoral dirigida por Marcela González y Julio Ogas, Departamento de Historia del Arte y Musicología, Universidad de Oviedo.

Ramos García, Ana María y Caurcel Cara, Ma Jesús (2011). Los podcasts como herramienta de enseñanza-aprendizaje en la universidad. Profesorado: Revista de currículum y formación del profesorado, 15/1, pp. 151-162.

Sánchez López, Virginia y Marín López, Javier (2009). Los conciertos didácticos del Festival de Música Antigua de Úbeda y Baeza: cinco años de experiencias educativas (20022006). Eufonía: Didáctica de la Música, 45, pp. 117-123. 
Solano Fernández, Isabel Ma y Sánchez Vera, Ma Mar (2010). Aprendiendo en cualquier lugar: el podcast educativo. Pixel-Bit. Revista de Medios y Educación, 36, pp. 125-139.

Turbau, Inma (2011). ¿Por dónde empiezo? Guía didáctica para programar, financiar y comunicar eventos culturales. Madrid: Ariel. 